\title{
IL FINANZIAMENTO DELLE PMI TRAMITE IL MERCATO DEI CAPITALI. IL RUOLO DI BORSA ITALIANA
}

\author{
Nota del s.c. ANDREA SIRONI (*)
}

(Adunanza del 25 ottobre 2018)

SuNTO. - Questo articolo esamina in modo sintetico le caratteristiche finanziarie delle imprese italiane, evidenzia l'importanza di un loro crescente accesso al mercato dei capitali per favorire gli investimenti, le politiche di internazionalizzazione e in ultima analisi lo sviluppo economico del Paese. Si descrivono inoltre le principali azioni adottate da Borsa Italiana negli ultimi anni per favorire questo processo. In chiusura si evidenziano anche le misure che sarebbe opportuno adottare a livello di politiche pubbliche per favorire questo processo virtuoso.

ABSTRACT. - This paper briefly describes the main fatures of the Italian companies financial structure and highlights the importance of a stronger access to the capital markets in order to rebalance their capital structure and finance their investments and their internationalisation strategies. The main actions adopted by Borsa Italiana to favor this process are also described in the second part of the paper. Finally, a number of measures that could be adopted by the policy makers are also briefly examined.

L'Italia è un Paese le cui imprese presentano una struttura finanziaria che si differenzia da quella delle imprese di altri paesi per due principali caratteristiche. Da un lato, la scarsa capitalizzazione, ossia la carenza di capitale di rischio rispetto al capitale di debito. Si tratta di

(*) Università Commerciale Luigi Bocconi, Milano, Italy.

E-mail: andrea.sironi@unibocconi.it 
una caratteristica che in parte riflette la forte prevalenza di imprese familiari. La seconda caratteristica è rappresentata da una prevalenza, nell'ambito del capitale di debito, di finanziamenti bancari di breve termine. In questo senso, le imprese italiane sono solite finanziarsi mediante prestiti bancari a breve scadenza e fanno invece scarso ricorso all'emissione di titoli nel mercato obbligazionari. Ne risulta una complessiva struttura finanziaria relativamente sbilanciata, caratterizzata da una carenza di risorse finanziarie stabili, a medio-lungo termine, e dunque una maggiore vulnerabilità rispetto a periodi negativi del ciclo economico e dunque della domanda.

Per questi motivi, la possibilità di raccogliere capitale - sia in forma di capitale di rischio, sia in forma di capitale di debito - risulta cruciale non solo per favorire la crescita dell'economia, ma anche per rendere le imprese italiane più resilienti rispetto a fasi di crisi. Nel corso degli ultimi anni si è attivato un circuito virtuoso che ha visto crescere sia gli investimenti nel capitale di rischio delle piccole e medie imprese (PMI) italiane, sia il numero di PMI che hanno scelto di aprire il proprio capitale azionario quotandosi in borsa.

Si tratta di un fenomeno importante che presenta diversi riflessi positivi. Da un lato, esso consente alle imprese di medie e piccole dimensioni, da sempre il punto di forza del sistema produttivo italiano, di rafforzare il proprio grado di patrimonializzazione e per questa via di aumentare gli investimenti e sostenere strategie di crescita interna ed esterna e percorsi di sviluppo internazionale.

Dall'altro, esso spinge le imprese verso una maggiore trasparenza, un miglioramento della governance aziendale e una maggiore capacità di attrarre una classe dirigente professionale e preparata. Un maggiore ricorso delle PMI al mercato dei capitali risulta inoltre particolarmente importante in un Paese come il nostro, dove oltre 1' $80 \%$ del finanziamento delle imprese proviene dal sistema bancario, in un contesto nel quale lo stesso sistema risulta vincolato nell'attività di prestito dai più stringenti requisiti di capitale introdotti negli ultimi anni dalle autorità di vigilanza.

Infine, la quotazione di un numero crescente di imprese consente di accrescere la dimensione del mercato borsistico italiano, storicamente caratterizzato da una capitalizzazione inferiore, in termini di rapporto con il PIL del paese, rispetto a quella degli altri principali mercati europei, in un contesto nel quale diversi studi empirici hanno evidenziato il contributo positivo alla crescita economica derivante dalla presenza di un mercato dei capitali liquido e sviluppato. 
A questa evoluzione positiva hanno contribuito sia fattori generali, quali il miglioramento della fase congiunturale e le favorevoli condizioni del mercato borsistico, sia fattori specifici, quali i piani individuali di risparmio (PIR) approvati dal governo con la legge di bilancio del 2017 per sostenere le piccole e medie imprese italiane, i quali prevedono importanti agevolazioni fiscali per gli investitori e alcune importanti innovazioni introdotte da Borsa Italiana negli ultimi anni, quali la piattaforma Elite, volta a favorire l'accesso al mercato delle PMI.

Più in dettaglio, vi sono oggi almeno tre fattori, fra loro collegati, che rendono vantaggioso il ricorso al mercato dei capitali. Il primo è rappresentato dall'elevata liquidità in cerca di interessanti opportunità di investimento. Oggi numerosi investitori, istituzionali e non, sono spinti dai bassi tassi di interesse a cercare investimenti che offrano maggiori rendimenti, anche a fronte di un maggior grado di rischio. Vi è in sostanza una domanda elevata a fronte di un'offerta limitata di opportunità di investimento. Si tratta di una situazione favorevole per un'impresa che intenda aprire al mercato il proprio capitale.

Infine, si tratta di una fase favorevole dal punto di vista fiscale, data l'introduzione dei Piani Individuali di Risparmio (PIR), i quali rappresentano un ulteriore fattore a favore dell'investimento in titoli azionari di PMI. Si tratta di un meccanismo che elimina la tassazione sui guadagni in conto capitale realizzati investendo - in un arco di cinque anni - in titoli azionari. E' previsto in particolare che una quota del portafoglio debba essere investita in titoli di imprese quotate che non fanno parte dell'indice principale (FTSE MIB), ossia di aziende di dimensioni non elevate. Questa evoluzione recente, che ha visto crescere gli investimenti in PMI quotate rappresenta una tendenza positiva. Occorre tuttavia che alla crescita dei fondi e della liquidità faccia seguito anche una crescita più significativa del numero di aziende di piccole e medie dimensioni quotate. Seppure il numero di società che hanno scelto di aprire il proprio capitale di rischio sia cresciuto negli ultimi mesi, esso resta ancora esiguo il numero di società quotate nel mercato italiano rispetto agli altri paesi europei più avanzati.

Infine, anche sul fronte della struttura finanziaria le imprese italiane presentano ancora un minore grado di patrimonializzazione. In questo senso, la raccolta di capitale di rischio rappresenta uno strumento efficace per ridurre la leva finanziaria, acquisendo risorse stabili che consentono di finanziare la crescita e i processi di internazionalizzazione. 
In questi ultimi anni Borsa Italiana ha avviato alcune importanti iniziative volte ad avvicinare le PMI al mercato. Esistono oggi differenti piattaforme che supportano le imprese nelle diverse fasi di sviluppo: dal primo stadio, con la piattaforma Elite, alla vera e propria espansione, con AIM Italia e - per la parte obbligazionaria - ExtraMOT, al consolidamento e all'espansione internazionale con il mercato principale (MTA) e il segmento dedicato alle aziende di eccellenza, lo STAR. L'iniziativa recente di maggiore rilevanza per quanto concerne le PMI è rappresentata da Elite. A fronte della riluttanza degli imprenditori italiani a quotarsi in borsa, è stato introdotto un percorso di avvicinamento, che non necessariamente conduce alla quotazione. Si tratta di un percorso formativo della durata di due anni che accresce la visibilità nazionale e internazionale dell'impresa, favorisce l'interazione con altre imprese e consente la raccolta di risorse finanziarie anche mediante strumenti di private placement. Ad oggi, hanno aderito oltre 600 imprese, di 25 paesi, che complessivamente presentano un fatturato di oltre 50 miliardi di euro e occupano oltre 200.000 persone.

Borsa Italiana ha peraltro di recente molto favorito lo sviluppo dello strumento delle SPAC, veicoli che consentono di portare aziende di qualità alla quotazione seguendo un percorso più graduale e meno incerto nell'esecuzione. Si tratta di particolari veicoli finanziari che raccolgono i fondi sul mercato e, in una fase successiva, si fondono con un'azienda target, la quale si trova automaticamente quotata. Dal 2011 ad oggi sono già state costituite 12 SPAC, le quali hanno condotto sul mercato aziende di grande qualità come Fila, Avio e LU.VE.

Vi sono almeno tre linee di azione che potrebbero essere adottate per consolidare e rafforzare la tendenza in atto. La prima è rappresentata da misure di agevolazione fiscale rivolte a investitori e imprese. L'introduzione dei PIR, con il connesso vantaggio fiscale sul lato investitori, rende più urgente l'adozione di misure volte a incentivare le imprese ad aprire il proprio capitale mediante la quotazione in Borsa. Il rischio è infatti che nel tempo possa determinarsi un eccesso di offerta di fondi in presenza di un numero troppo esiguo di società quotate, ossia di opportunità di investimento. Una prima soluzione è rappresentata dalla defiscalizzazione, per le imprese che decidono di quotarsi, dei connessi costi di quotazione (advisor, spese legali, commissioni, ecc.) mediante ammortamento accelerato, iperammortamento o credito d'imposta. Una seconda soluzione è rappresentata dall'introduzione di una sorta di ACE maggiorata per gli aumenti di capitale realizzati attra- 
verso la Borsa, ossia una maggiorazione dell'aumento di capitale a cui applicare l'aliquota figurativa dell'ACE.

Sul fronte investitori in realtà l'introduzione dei PIR ha già fornito un incentivo forte, come visto sopra, all'investimento in PMI quotate. Il rischio, tuttavia, è che questa misura, limitata nel tempo, non produca l'effetto sperato di favorire lo sviluppo duraturo di una classe di investitori professionali specializzati in una categoria di investimenti, le PMI, che, pur essendo al centro delle politiche di sviluppo del Paese, rimane ancora residuale nei portafogli degli investitori istituzionali. In questo senso, estendere agli investitori previdenziali la possibilità di investire in PIR o ancora favorire lo sviluppo di un "Fondo di Fondi" per le PMI quotate potrebbe risultare funzionale all'obiettivo di consolidare una base di investitori dedicati alle PMI.

Una seconda linea di azione riguarda le iniziative di comunicazione e promozione della quotazione in Borsa. Uno dei principali ostacoli a un pieno sviluppo del mercato dei capitali nel nostro Paese è rappresentato dalla riluttanza di molti imprenditori, i quali sovente controllano imprese di natura familiare da generazioni, all'apertura del capitale. Questo atteggiamento trova fondamento in diversi fattori: il timore che l'apertura del capitale si associ all'introduzione di controlli e burocrazia, l'avversione nei confronti di una maggiore trasparenza che inevitabilmente si associa all'ingresso di nuovi soci, il timore di perdere il controllo e di non poter così gestire l'azienda, sovente in modo non nettamente distinto dalla famiglia, con la tradizionale flessibilità.

Con riferimento a quest'ultimo aspetto, è opportuno osservare come la recente introduzione del meccanismo del voto multiplo in Italia consenta di raccogliere capitale di rischio senza necessariamente diluire il controllo. Con riferimento agli altri aspetti, vale la pena di osservare come la crescita e lo sviluppo di un'imprese debbano necessariamente passare per una netta distinzione fra le attività dell'impresa e quelle della famiglia, una maggiore trasparenza, lo sviluppo di una governance adeguata e coerente con le migliori prassi. Si tratta di aspetti che si rendono necessari non solo per raccogliere capitali sui mercati, ma anche per poter attrarre e ritenere risorse umane professionali e qualificate.

Più in generale, occorre che alla quotazione in borsa e, più in generale, alla raccolta di capitale sul mercato, vengano associati quei valori positivi che di fatto caratterizzano questo canale di finanziamento delle imprese e degli investimenti produttivi: trasparenza, stabilità delle 
fonti di finanziamento, costo contenuto, possibilità di sostenere percorsi ambiziosi di crescita e di internazionalizzazione.

Per sostenere questa seconda linea di azione sono necessarie iniziative di comunicazione promosse dal Governo, dagli opinion leader in ambito imprenditoriale e dai media in generale, che associno aspetti valoriali positivi allo status di azienda quotata. In questa direzione negli ultimi mesi già si stanno attivamente muovendo sia il Ministero dell'Economia e della Finanze, sia Confindustria. Occorrono inoltre iniziative concrete di origination promosse dagli operatori del settore, dalle banche, dai fondi di private equity. Anche su questo fronte, specie dopo l'introduzione dei PIR, sono state avviate numerose iniziative.

Infine, una terza linea di azione riguarda le autorità di vigilanza e la Consob in particolare. Su questo fronte, sarebbe auspicabile che i processi di quotazione sul mercato regolamentato venissero snelliti e ottimizzati e che le relative tempistiche venissero rese più rapide e certe, avendo consapevolezza del ruolo positivo per l'economia del Paese che assume la raccolta di capitali sul mercato delle imprese.

Il nostro Paese sta vivendo una fase positiva di ampliamento e apertura del mercato dei capitali. Alcune iniziative promosse negli anni passati dal Governo hanno favorito l'afflusso di capitali al mercato. Parallelamente, importanti iniziative promosse dall'industry e dalla Borsa hanno avvicinato numerose imprese di qualità, caratterizzate da ambiziosi piani di crescita, al mercato. Si tratta di imprese di diversi settori, rappresentative del tessuto produttivo del Paese, sopravvissute a una crisi finanziaria profonda e duratura. Un rafforzamento della loro struttura finanziaria attraverso l'apertura al mercato dei capitali, l'adozione di una governance allineata alle best practice internazionali e il finanziamento di piani di crescita e sviluppo internazionali non va solo a beneficio delle singole imprese, ma dell'intero sistema economico italiano. Occorre dunque potenziare gli sforzi, non solo dell'industry, ma anche dei policy makers e delle autorità di vigilanza, affinché questo recente trend positivo si consolidi e prosegua anche nei prossimi anni. 